\title{
What is Digital Health? Review of Definitions
}

\author{
Farhad FATEHI $^{\mathrm{a}, \mathrm{b}}$, Mahnaz SAMADBEIK ${ }^{\mathrm{c}, 1}$ and Azar KAZEMI ${ }^{\mathrm{d}}$ \\ ${ }^{a}$ Monash University, Melbourne, Australia \\ ${ }^{\mathrm{b}}$ Tehran University of Medical Sciences, Tehran, Iran \\ ${ }^{c}$ Lorestan University of Medical Sciences, Khorramabad, Iran \\ ${ }^{\mathrm{d}}$ Mashhad University of Medical Sciences, Mashhad, Iran
}

\begin{abstract}
Digital technologies are transforming the health sector all over the world, however various aspects of this emerging field of science is yet to be properly understood. Ambiguity in the definition of digital health is a hurdle for research, policy, and practice in this field. With the aim of achieving a consensus in the definition of digital health, we undertook a quantitative analysis and term mapping of the published definitions of digital health. After inspecting 1527 records, we analyzed 95 unique definitions of digital health, from both scholar and general sources. The findings showed that digital health, as has been used in the literature, is more concerned about the provision of healthcare rather than the use of technology. Wellbeing of people, both at population and individual levels, have been more emphasized than the care of patients suffering from diseases. Also, the use of data and information for the care of patients was highlighted. A dominant concept in digital health appeared to be mobile health (mHealth), which is related to other concepts such as telehealth, eHealth, and artificial intelligence in healthcare.
\end{abstract}

Keywords. digital health, definition, term mapping, content analysis

\section{Introduction}

Digital Health is an emerging field of study at the intersection of healthcare and digital technologies, which has attracted lots of attention in the past decade in many countries around the world. In 2019, the American Medical Association reported that companies have invested billions of dollars on new digital health entrepreneurship [1]. The US Food and Drug Administration considers a broad scope of technologies as digital health; mobile health, wearable devices, telehealth and telemedicine, health information technologies, and personalized medicine [2]. WHO emphasizes that digital health can be beneficial to achieving the Sustainable Development Goals by making health and wellbeing services accessible with high standards for all people globally [3].

The term "digital health" is broadly used in the various disciplines such as health informatics, but there is no agreed upon definition for this term. Due to different perspectives of academia, scientific institutions, industry, and individuals, there is a lack of comprehensive and precise definition of digital health. A systematic review of the literature identified the following components of digital health innovation ecosystem: e-

\footnotetext{
${ }^{1}$ Corresponding Author, Mahnaz Samadbeik, School of Allied Medicine, Lorestan University of Medical Sciences, East Goldasht, Khorramabad, Iran. Postcode: 6819789741; Email: mahbeik@yahoo.com.
} 
health, m-health, health 2.0, telehealth and telemedicine, public health surveillance, personalized medicine, health promotion strategies, self-tracking, wearable devices and sensors, genomics, medical imaging, and information systems [4]. According to the Health Informatics Society of Australia and Digital Health Workforce Academy paper, digital health uses not only electronic data but also traditional data to serve healthcare and research. Today, artificial intelligence and machine learning are used as essential methods in digital health scope, to combine with ICT and other technologies to solve consumer and patients' problems [5].

The first reference to digital health in PubMed database dates back to 90s, when this concept was mainly used for digitization of health information and libraries [6]. In the 2000 s, with the spread of the Internet worldwide, the concept of digital health changed. Later on, with the advancement of computer science and informatics, and their applications in health care, a number of new concepts such as artificial intelligence and genomics were also considered as part of digital health. However, ambiguity in the definition of digital health and its taxonomy remains yet to be addressed. There is, therefore, a need to consolidated digital health concepts for use in research, policy, and practice. We envisaged to reach a consensus definition for digital health that can satisfy most, if not all, of the stakeholders. In this study we collected, examined, and quantitatively analyzed the published English definitions of digital health both in scholarly literature and online sources.

\section{Methods}

We conducted a term map analysis of the published definition of 'digital health' using VOSviewer software version 1.6.15 (Centre for Science and Technology Studies, Leiden, The Netherlands) [7]. The definitions were obtained from two sources: 1) peerreviewed publications, and 2) websites of relevant authorities and scientific bodies. We first searched Web of Science, PubMed, Scopus, and Google Scholar using this search string: "digital health" AND (definition OR defined). The publication period was between January 2000 and April 2020 (last search, 15 May 2020), limited to publications in English. Furthermore, we searched Google using two search queries in two separate runs: A) "digital health" AND (definition OR defined); B) "What is digital health". As Google ranks the retrieved websites based on their importance and relevance, we reviewed the first 200 results for each of these two searches.

This study included all resources (articles, reports, letters, guidelines, discussion papers, and websites) that have defined or attempted to define digital health in explicit terms. Documents were excluded if they did not provide an original definition, or they focused on the other aspects of digital health (e.g. digital health technology, digital health frameworks, and digital health interventions) rather than its definition. The search results were exported to EndNote, and duplicates were removed. Two authors independently assessed the titles, abstracts, full texts, and websites for eligibility. Disagreements about study eligibility were resolved through consensus discussion or by consulting the third author. The final set of selected references was then reviewed, and the definitions were extracted for term mapping. From the selected references, we extracted the following data: author name, publication year, title, source, URL, and the definition.

We carried out a quantitative analysis to find the most common terms used in the included definitions. VOSviewer was used to visualize the main terms and concepts in the digital health definitions. A thesaurus file was used to perform data cleaning and 
merging terms in VOSviewer (e.g. "mhealth", "m health", and "Mobile health" were merged as "Mobile health"). We excluded a number of general terms that were commonly used, but did not add value to the definition of digital health, such as "definition", "term", and "field". We used the full counting method of occurrence of terms in the VOSviewer software. For the term occurrence map, terms that appeared in at least three of the definitions (threshold level of terms equal to 2) were selected, so terms with fewer than 3 occurrences are excluded.

\section{Results}

We screened 1,527 sources ( 855 peer-reviewed articles and 672 web pages), which were returned by our scholar and general electronic search. After inspecting the contents of these references, we extracted 95 unique definitions of "digital health" in this study (30 definitions from journal articles and 39 from websites). The list of included articles and websites is available at https://osf.io/yfusw/.Total main terms included in the definitions were 410, and 60 terms were repeated at least 3 times. Table 1 lists the top ten terms with the highest frequency in the included definitions. Terms with a high relevance score tend to represent specific topics covered by the text data, including "disruptive technology" (6.25), "human health" (3.46), and "healthcare service" (2.49). However, terms with a low relevance score tend to be of a general nature, including "health" (0.09), "use" (0.12) and "technology" (0.18).

Table 1. Ten top terms with the highest frequency in the definitions

\begin{tabular}{|l|c|c|}
\hline \multicolumn{1}{|c|}{ Term } & Occurrence & Relevance score \\
\hline Health & 49 & 0.09 \\
\hline Technology & 35 & 0.18 \\
\hline Use & 31 & 0.12 \\
\hline Information & 25 & 0.35 \\
\hline Mobile health & 24 & 0.53 \\
\hline Healthcare & 19 & 0.31 \\
\hline Medicine & 14 & 0.71 \\
\hline Wellness & 14 & 0.48 \\
\hline Patient & 14 & 0.46 \\
\hline eHealth & 13 & 0.42 \\
\hline
\end{tabular}

To visualize the most frequent terms used in the definitions, a network map and a density map of the terms were created. The terms included in the map are selected based on the calculation of occurrences and relevance scores. According to the map of words (Figure 1), among the terms that met the threshold, the terms "health," "technology", and "use" were occurred the most in the definitions of digital health. Six major clusters emerged and were classified according to the 52 most common terms with an occurrence of at least three times.

Figure 2 shows the terms density in 95 definitions visualized by VOSviewer. The bubble size indicates the number of definitions containing each term. If the terms frequently co-appeared in the same definitions, their bubbles would be closer to each other. The color of each point in this visualization indicates the density of items at that point. By default, colors range from blue to green to yellow. The yellow color of the point shows the larger number and the higher weights of items in its neighborhood, which in this case were the terms "health", "technology", and "use". The blue color of the point shows the smaller number and the lower weights of items in its neighborhood, such as the terms "health service", "health risk", and "illness". 


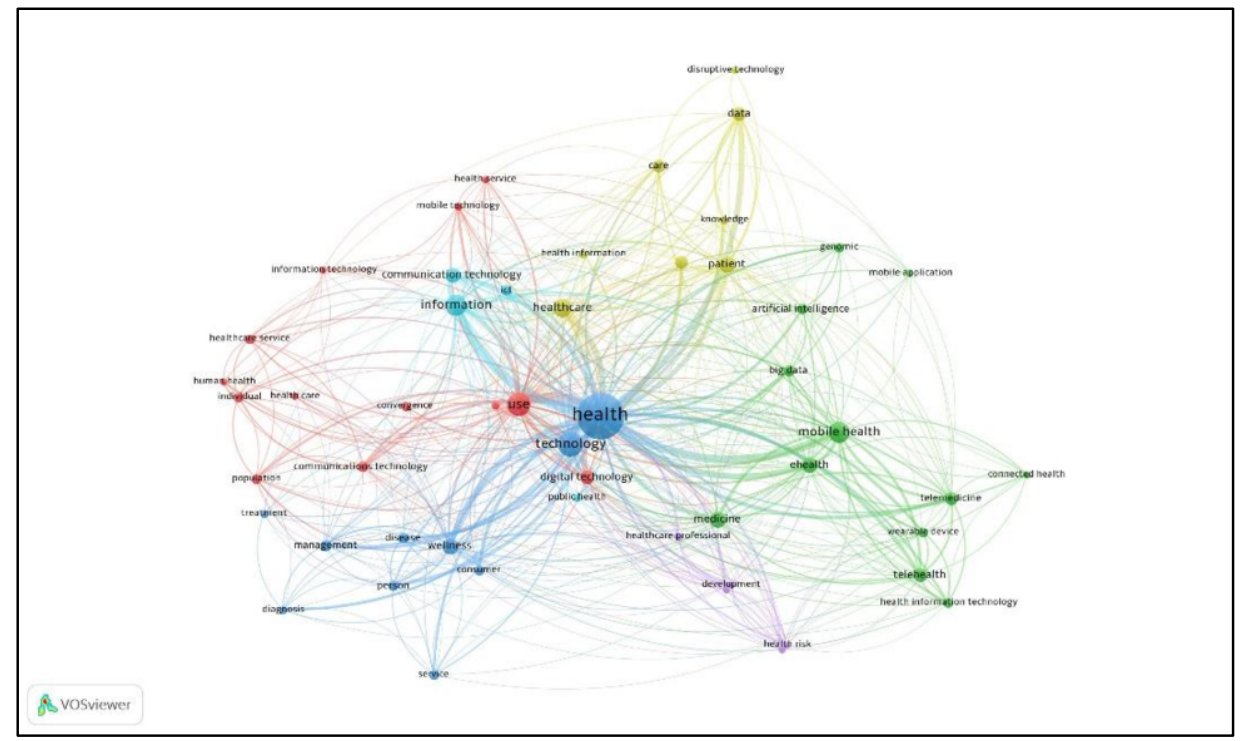

Figure 1. Terms occurrence network in the 95 definitions of digital health visualized by VOSviewer

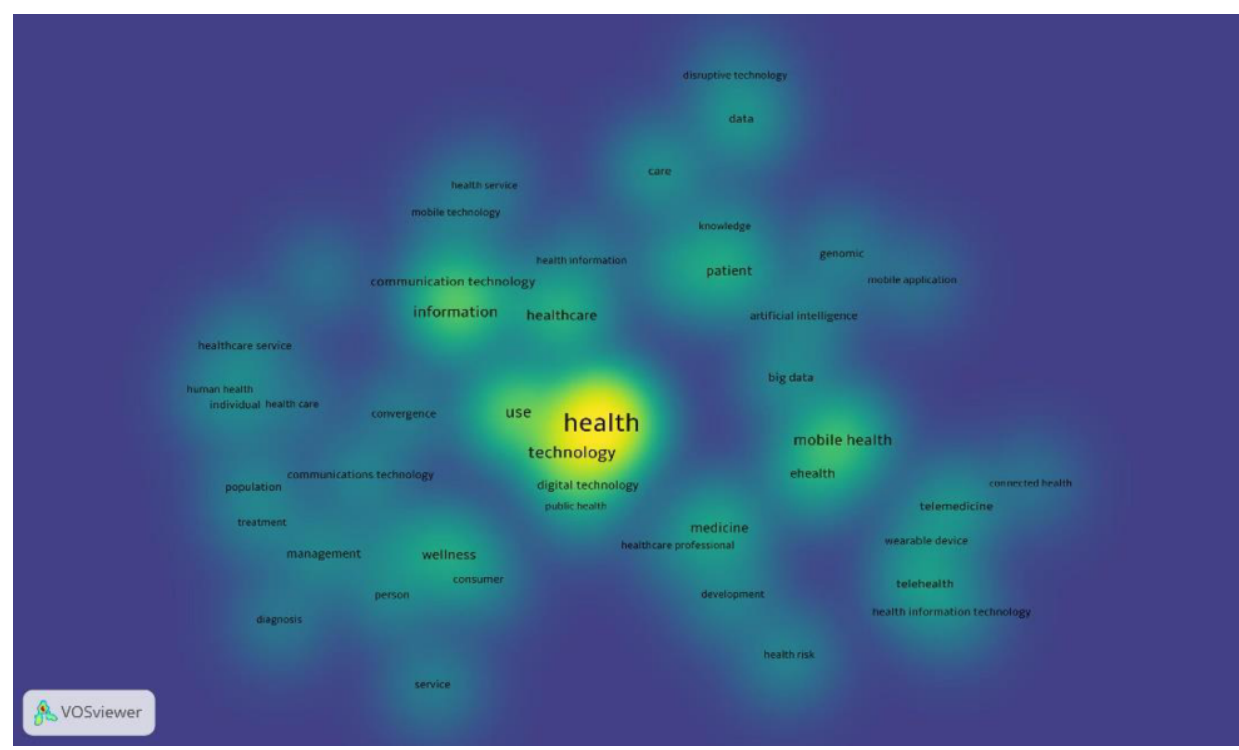

Figure 2. Terms density visualization of the 95 definitions of digital health. 


\section{Conclusion}

We reviewed, quantitively analyzed, and mapped the main terms of 95 unique definitions of 'digital health'. The results of this study show that in the field of digital health, the main focus is on the health, rather than technology. Under the concept of health, emphasis was on the health and wellbeing of individuals and population, rather the diseases and patients. It is evident that in the concept of technology, emphasis is on the (proper) use of technology, rather than its technical aspects. Mobile Health (mHealth) appeared to be a dominant concept in the field of digital health, and closely related to artificial intelligence and through it connected to genomics. Moreover, the concepts of data and information, were closely related to patients. Based on the results of this study, we can infer that digital health is about the proper use of technology for improving the health and wellbeing of people at individual and population levels, as well as enhancing the care of patients through intelligent processing of clinical and genetic data.

\section{References}

[1] O'Reilly KB. Digital health funding hits $\$ 8.1$ billion. How to spend it wisely [Internet]. 2019 [cited 12 Sep 2020]. Available from: https:/www.ama-assn.org/practice-management/digital/digital-healthfunding-hits-81-billion-how-spend-it-wisely.

[2] US Food and Drug Administration (FDA). Digital health innovation action plan [Internet]. 2019 [cited 12 Sep 2020]. Available from: https://www.fda.gov/medical-devices/digital-health.

[3] World Health Organization. Digital Health [Internet]. 2020 [cited 12 Sep 2020]. Available from: https://www.who.int/health-topics/digital-health

[4] Iyawa GE, Herselman M, Botha A. Digital health innovation ecosystems: From systematic literature review to conceptual framework. 2016.

[5] Rowlands D. What is digital health? And why does it matter? [White paper]. Health Informatics Society of Australia 2020. Available from: https://www.hisa.org.au/wpcontent/uploads/2019/12/What_is_Digital_Health.pdf

[6] Galvin JR, D'Alessandro MP, Erkonen WE, Smith WL, El-Khoury GY, Weinstein JN. The virtual hospital. Providing multimedia decision support tools via the Internet. Spine. 1995;20(15):1735-8.

[7] Van Eck NJ, Waltman L. Software survey: VOSviewer, a computer program for bibliometric mapping. Scientometrics. 2010 Aug;84(2):523-38. 